\title{
Use the Brute_Force Pattern Matching Algorithm for Misuse Intrusion Detection System
}

\author{
Haleema Essa Sulaiman \\ haleema_essa@uomosul.edu.iq \\ College of Computer Sciences and Mathematics \\ University of Mosul, Mosul, Iraq
}

Received on: 19/07/2018

Accepted on: 24/01/2019

\section{ABSTRACT}

Security issues, like network intrusion and viruses, have been increased widely with the growth of computer applications and networks. Therefore, it becomes necessary to develop methods to protect information from malicious attacks within the different environments. One of these methods is to use intrusion detection system for the detection of different interventions.

The research was presented a way to detect misuse intrusion (Misuse Detection System), as was performed classification of events, which will be either the events of Normal Events or Intrusion Events. This classification process has been based on one of the String Pattern matching Algorithms, which is Brute_Force algorithm.

Brute_Force algorithm is used after making a comparison between this algorithm and another two algorithm (Knuth - Morris - Pratt String Matching and Boyer-Moore Algorithm).

Data processed in the work is taken from the KDD list. The written version of this data, which will be similar to the data format in the comma separated values files (CSV), This data has been converted to tables and then a comparison between these tables is made for the purpose of categorizing events based on the algorithm mentioned above. Java language has been used in this work as one of the most powerful programming languages, has been the adoption of Eclipse environment to write Java classes used in the work.

Keywords: intrusion detection, misuse intrusion detection, string pattern matching algorithms, tables comparison, events classification, java language.

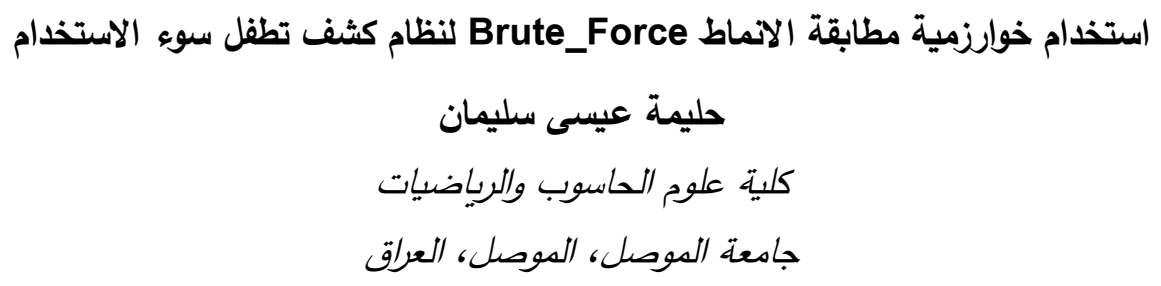

تاريخ القبول: 2019/01/24

تاريخ الاستلام: 2018/07/19

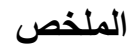

قدم البحث طريقة لكثف سوء الاستخدام ( Misuse Detection System )، إذ اجري تصنيف

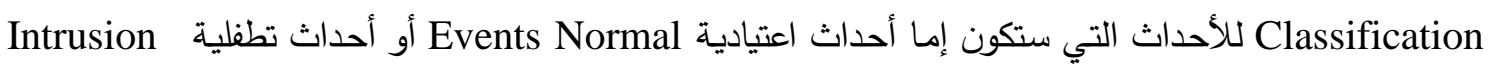

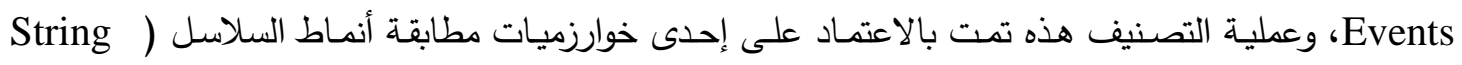

Prute_Force Pattern matching Algorithms

اسنخدمت هذه الخوارزمية في النظام المقترح كونها الأكفأ عند التعامل مع بيانات النظام مقارنة بخوارزمية

( Knuth - Morris - Pratt String Matching ) 
البيانات التي تم التعامل معها في العمل مأخوذة من بيانات KDD، وقد استقيد من الصيغة المكتوبة بها

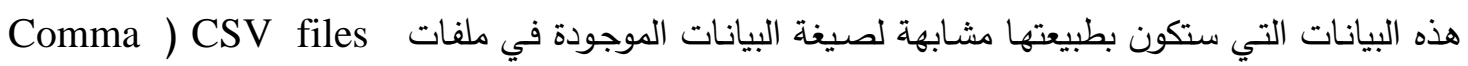
هدseparated Values Files الجداول لغرض القيام بعطية تصنيف الأحداث بالاعتماد على الخوارزمية المذكورة سابقاً.

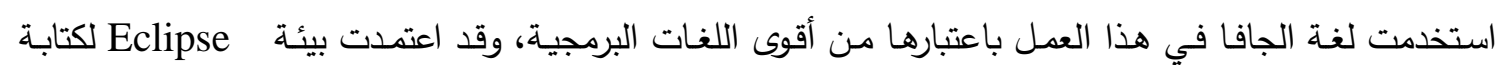
أصناف الجافا المستخدمة في العمل. الكلمـات المفتاحيـة: كثف تطفل، كثف سوء الاستخدام، خوارزميات مطابقة انماط السلاسل، مقارنـة الجداول، تصنيف الاحداث، لغة الجافا.

1 - مقدمة

إن تعقيد أنظمة الحواسيب الموزعة وأهميتها وموارد المعلومات المتوافرة فيها تمت بسرعة كبيرة، استتاداً

إلى هذه الحقيقة فقد أصبحت الحواسيب وشبكاتها هدفاً لجرائم الحاسوب التي ازدادت أكثر فأكثر [1].

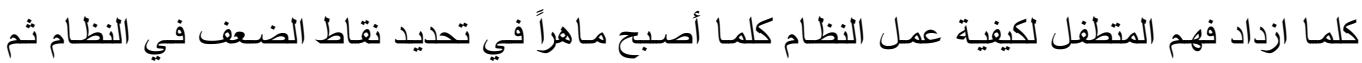
يستغلها للحصول على مستوى شرفية عالٍ الذي يستطيع بـه أن يسيطر على النظام. إن المتطفلين يستخدمون أنماطاً من التطفل التي من الصعوبة تحديدها أو مراقبتها [2]. استخدمت بعض التقانات لحماية البيانات المهمة مثل الجدار الناري والتشفير والتخ، إن أي تقانة أمنية

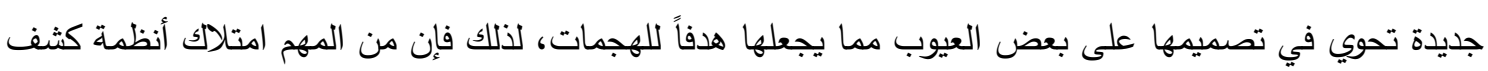

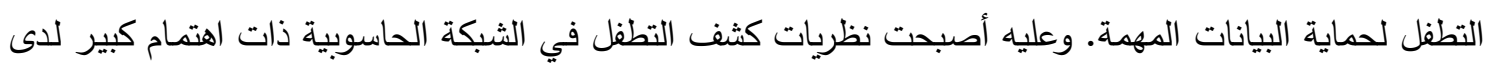
العديد من الباحثين في السنوات الأخيرة [3]. نظام كثف التطفل Intrusion Detection System) مكون مهم في إطار أمنية الحاسبة والمعلومات، وإن هدفه الرئيس هو تمييز بين الفعاليات الاعتيادية للنظام والسلوكيات التي ممكن أن نصفها على فئى إنى أنها تطفل، إن أي نظام لكثف التطفل لا يهمل استخدام التقانات الأمنية الأخرى ولكن يعمل كخط دفئ دفاع أخئ أخير في النظام [3].

يجهز (IDS) فائدتين أساسيتين هما الوضوحية (Visibility) والسيطرة ( Control ) ) وفي حالة دمج

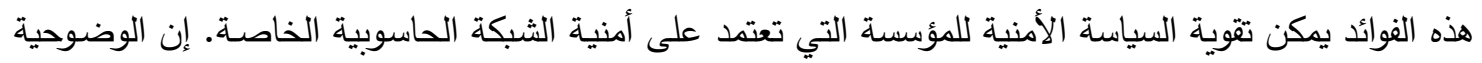

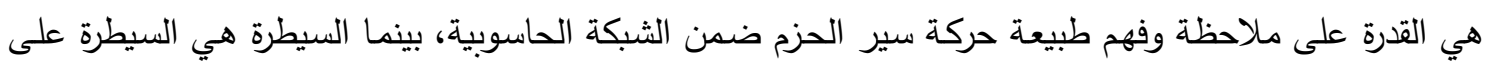

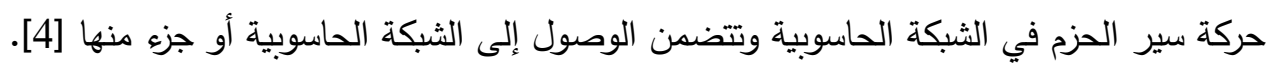

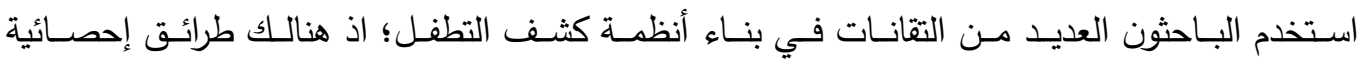

(5rtificial Intelligence Techniques ) وتقانات ذكائية اصطناعية (Statistical Approaches)

تستخدم خوارزميات مطابقة الأنماط ( Pattern Matching Algorithms ) لحل المشاكل المعقدة التي

Linear تكون غير قابلة للحساب أو التحليل المباشر وتطبيقات هذا المجال تشمل خوارزميات البحث المتسلسل

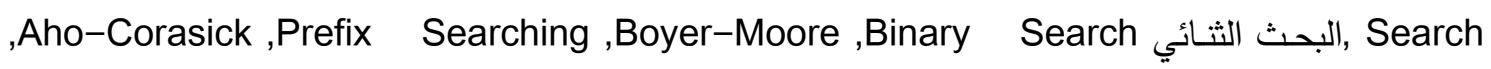
Soundix Searching 
استخدمت خوارزمية مطابقة أنماط السلاسل (Brute_Force String Pattern Matching Algorithm ) في عمليـة كثف التطفل وتصنيفه، واستخدمت لغـة الجافـا في هذا البحث، وبالاعتمـاد على الجداول التي كونـت باستخدام الصنف JTable ادخلت البيانات التي حصل عليها من مجموعة بيانات KDD CUP 99 التي تعتبر الأكثر استخداماً في تقييم نظم كثف التطفل. يمكن إيجاز أهم الأهداف للبحث بالنقاط الآتية:

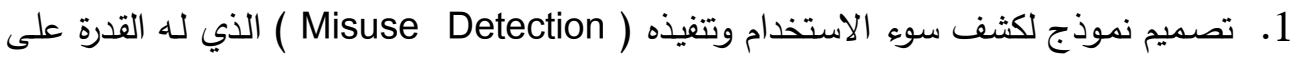

$$
\text { تصنيف مجموعة أحداث النظام إلى صنفين حدث اعتيادي أو حدث تطفلي. }
$$

2. إيجاد طريقة سهلة للتعامل مع ملفات (comma separated value files) (csv files)

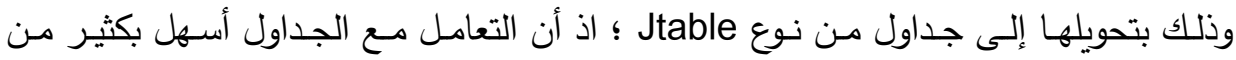
التعامل مع بيانات تعتمد مثل هذه الصيخ.

3. استخدام خوارزمية مطابقة الأنماط Brute Force لغرض تصنيف الاحداث، إذ استخدمت

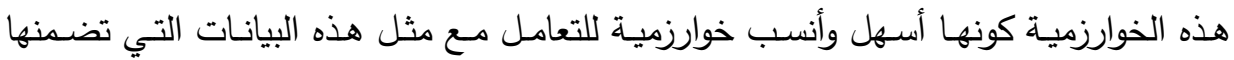

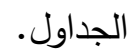
4. استخدام الأدوات الأفضل والأكفأ لإجراء عملية المقارنة التي تتمثل باستخدام لغة الجافا والتي شغلت باستخدام بيئة eclipse لتنفيذ الانموذج الامني.

\section{2- نظام كشف التطقل Intrusion Detection System}

تواجه جدران النار عدداً من نقاط الضعف منها أنها عملية (نعم أو كلا)، وهذا يعني أن ما يقوم به جدار النار هو منع المتطفل من الدخول إلى نظام الهدف، وأنه يعمل على فتح منفذ (Port) محدد فقط، في حين يغلق كل المنافذ

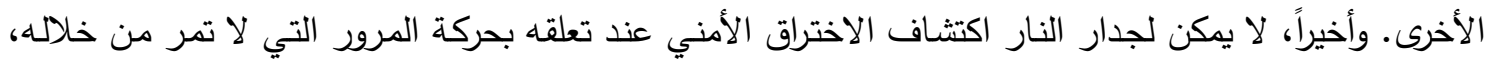

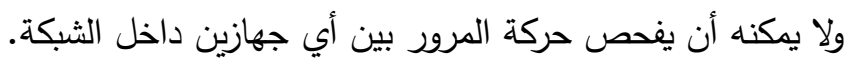
وتعد نقاط الضعف المذكورة سابقاً محفزات باتجاه استخدام آلية أمنية أخرى تعمل على دئ الثبكة، ويتوجب دانب أن تكون أكثر ذكاءً من جدار النار ؛ إذ إنها ترفع تحذيراً لأي متطفل وتكثف عن الحزم كلها في الثبكة في وقت حقيقي،

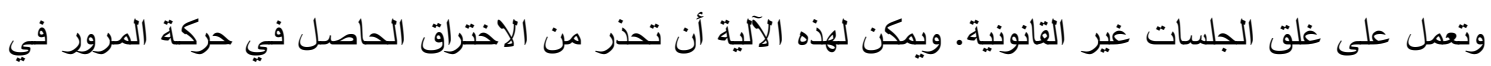

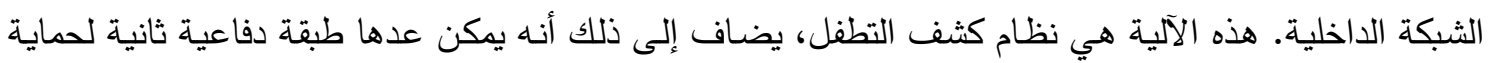

الشبكة [7]. وتُعرف أنظمـة كثف التطفل بأنها المُكون الذي يقوم بتحليل عمليات النظام وعمليات المستخدم في الحاسوب وأنظمة الشبكة للبحث عن نشاطات غير مرغوب فيها من وجهة نظر أمنية. وتُعد طريقة نظام كثف التطفل طريقة

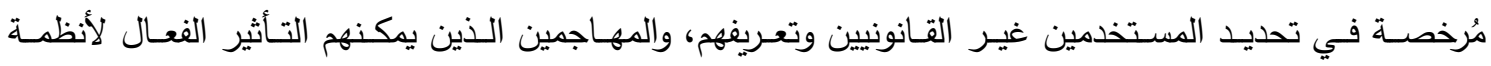
الحاسوب. وتقوم أنظمة كثف التطفل بالكثف عن عدد من التطفلات وتتفذ قسماً من الأعمال المحددة مسبقًا عندما يتم الكثف عن التطفل [8]. وأنظمـة كثف التطفل عبارة عن منتجات من البرامج الحاسوبية أو المعدات الحاسوبية التي تعمل على مراقبة العملية وتحليلها أوتوماتيكياً. ويعمل أي نظام للكثف عن التطفل على التحقق من أي نشاط متجهـ إلى داخل 
الثبكة أو خارجها، وسجلات النظام والأحداث، وتحديد النماذج المشتبه بها أو الأحداث التي قد تثير إلى وجود هجوم على النظام أو الشبكة من شخص ما يحاول الدخول عنوة أو يعمل على تخريب النظام.

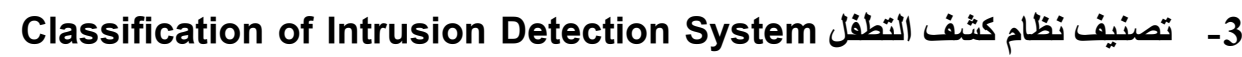

هناك عدة تصنيفات ودراسات متلاحقة للكثف عن التطفل. وعلى الرغم من هذه الجهود السابقة حتى

الوقت الحاضر فإن الكثف عن التطفل لايزال يفتقر إلى تصنيف وقابلية للتطبيق [8,9]. ويبين الثكل (1) تصنيف نظام أنموذجي للكثف عن التطفل.

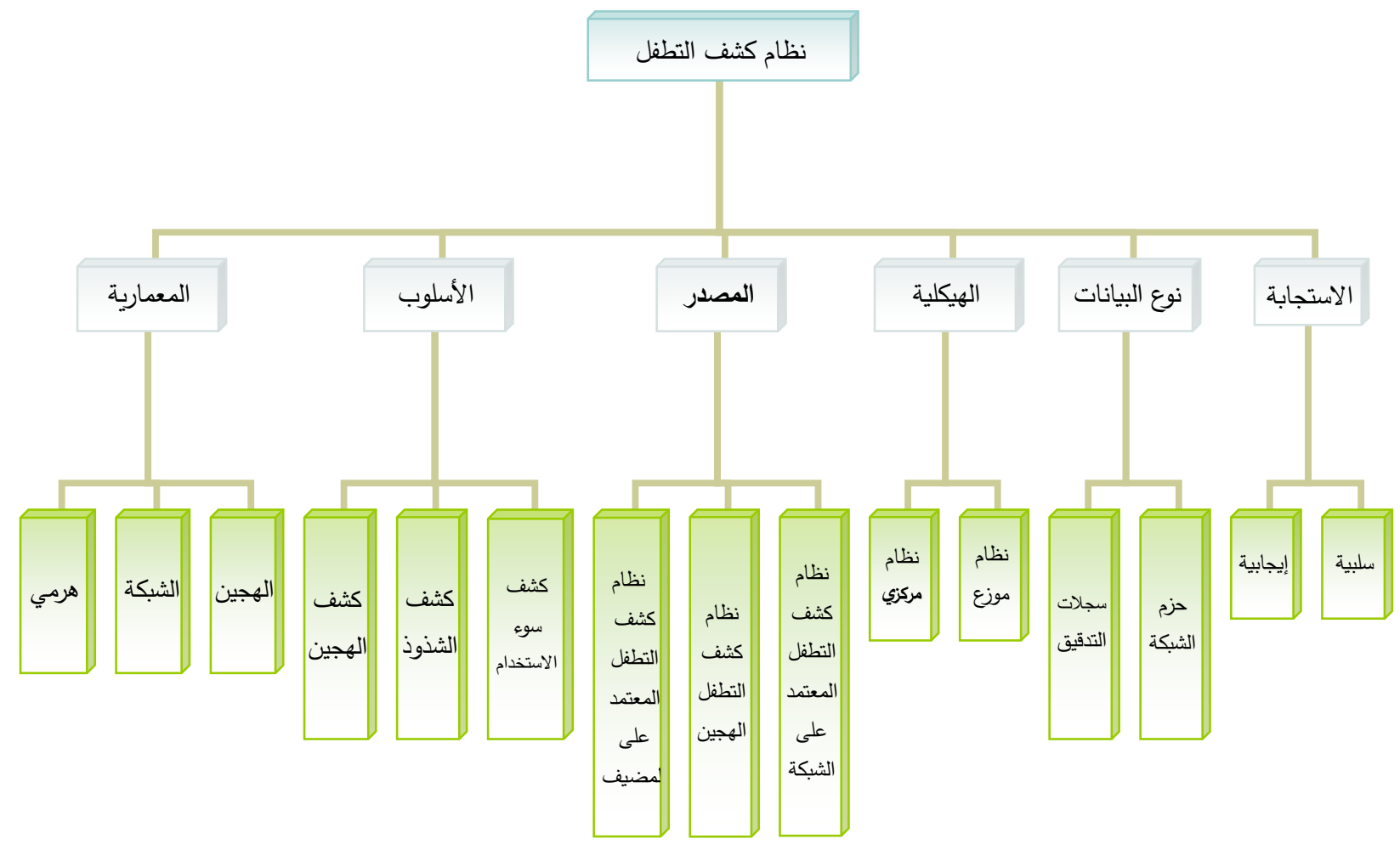

الثكل (1): تصنيف نظام كثف التطفل [9].

Misuse detection - كثف إساءة الاستخدام

يشير كثف إسـاءة الاستخدام إلى التقانات التي تميز أسـاليب معروفة لاختراق النظام. ويمكن وصف هذه

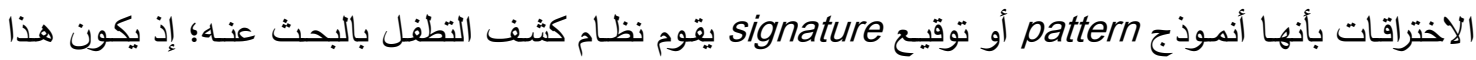
الأنموذج أو التوقيع عبارة عن سلسلة ثابتة أو مجموعة من الإجراءات وتستتد استجابات النظام على الاختراقات

$$
\text { التي تم تحديدها[10]. }
$$

هناك أربع مراحل رئيسة لتحليل عملية كثف إساءة الاستخدام:

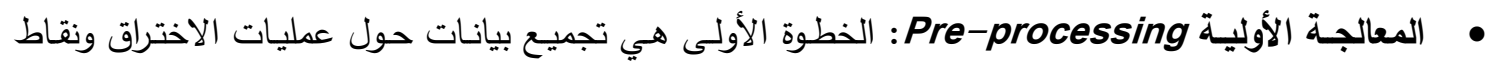

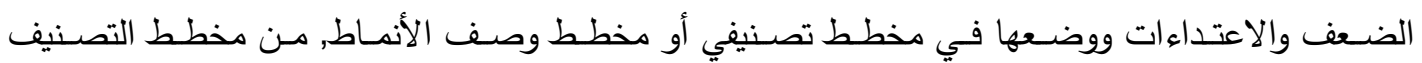
الأنموذج السلوكي و يوضع بعد ذلك في شكل محدد. 
Analysis التحليل Analysis : تقارن بيانات السلوك مـع قاعدة المعرفة باستخدام محرك التحليل

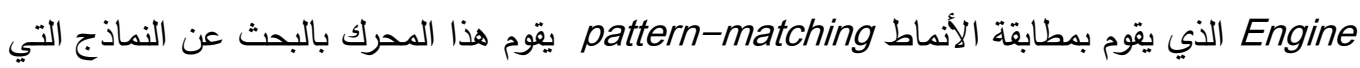
يتم اعتبارها هجمات. الاستجابة Response : إذا تطابقت بيانات السلوك مع نماذج الهجمات يقوم محرك التحليل بإرسال تنبيه إلى مسؤول النظام. • دقة تطابق البيانات Accuracy : إن دقة تطابق البيانات pattern-matching تعتمد على تحديث

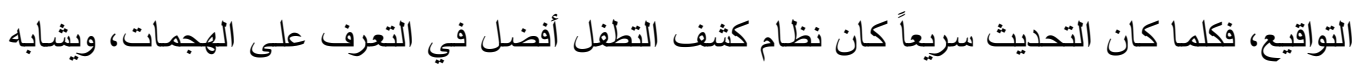
في هذه الصفة البرامج المضادة للفيروسات Antivirus. وتسمح أنظمة كثف التطفل فئل بالتحديث اليدوي أو الأوتوماتيكي للتواقيع الخاصة بالهجوم [11]. هناك ثلاثة أساليب مختلفة للكشف الخاص بإساءة الاستخدام: 1- مطابقة النمط Pattern-matching : تحديد الهجمات عن طريق عمليات فحص مرور البيانات

$$
\text { وتحديد توقيع نماذج الهجمات المعروفة. }
$$

2- تحليل البروتوكول Protocol analysis : التدقيق يكون أيضا عبر مراقبة مرور البيانات ومشاهدة

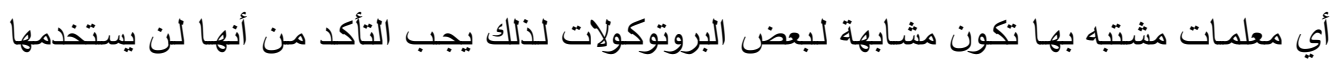
المهاجم بطريقة خبيثة malicious. طريقة التخمين Heuristic method : تكون هذه النظريـة مقاربـة لنظريـة كثف الفيروسـات في الأنظمـة المضادة للفيروسات Antivirus systems [11,12]. من محاسن أنظمة كثف إساءة الاستخدام أن التحليل الذي يستخدم في مطابقة الأنماط يكون أكثر كفاءة من تقانة كثف الثذوذ بسبب عدم وجود حسابات للقياسات الإحصائية، ولكن في المقابل هنالك بعض المسناوئ

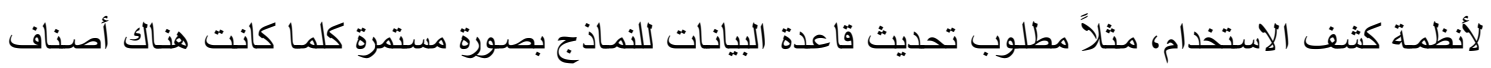

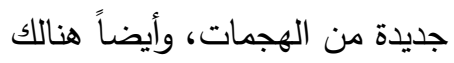

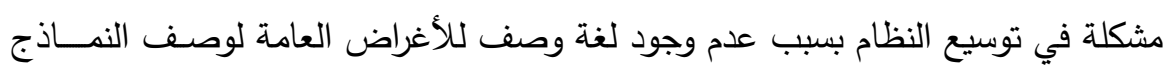

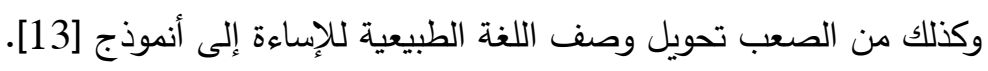

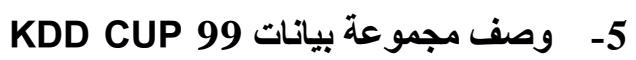

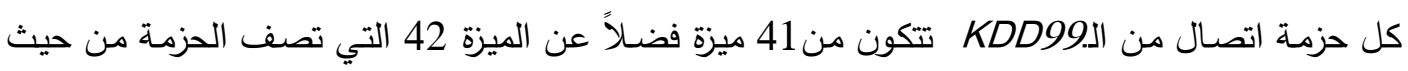

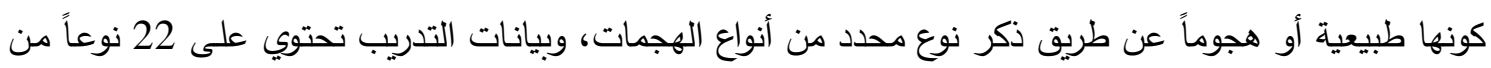

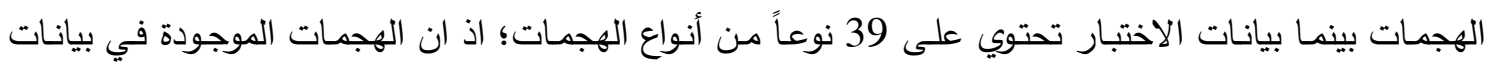

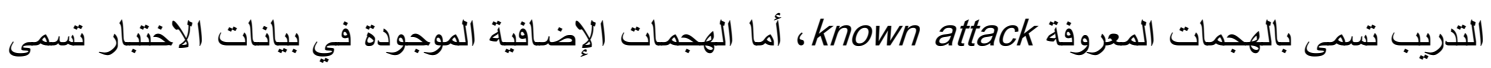
بالهجمات الجديدة novel attacks وتكون غير متوافرة في بيانـات التدريب[14]. والجدول رقم (1) يوضـح الهجمات المعروفة والهجمات الجديدة. الهجمات جميعها سواء أكانت معروفة أم جديدة تقع في واحدة من الفئات الأربعة الآتية: 1. Denial of Service Attack (DoS) : هجوم منع الخدمة أو الحرمان من الخدمة 
هو أن يقوم المهاجم بعمل بعض الحسابات وجعل المصادر مشغولة جدا أو جعل الذاكرة ممتلئة أو منع وصول المستخدمين القانونيين إلى الحاسبات.

2. Probing Attack : هو محاولة لجمع معلومات من أجهزة الحاسوب لمعرفة نقاط الضعف في

الحاسبة والتحايل عليها.

3. User to Root Attack (U2R) يقوم المهاجم باستغلال المستخدم العادي للنظام حيث يدخل ولن

النظام بصلاحيات المستخدم العادي مثلاً عن طريق الحصول على كلمة السر ومن ثم استغلال بعض لمض لمان

نقاط الضعف للوصول إلى جذر النظام.

4. Remote to Local Attack (R2L) : تكون للمهاجم القدرة على إرسال حزم إلى حاسبة من خلال

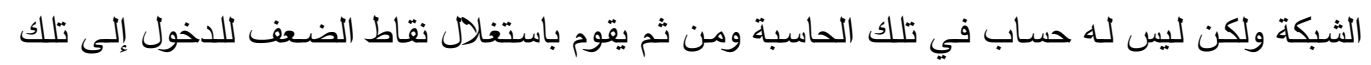

الحاسبة بوصفه مستخدم طبيعي لها [14,15].

\begin{tabular}{|c|c|c|c|}
\hline DOS & PROBE & $\mathrm{R} 2 \mathrm{~L}$ & U2R \\
\hline \multicolumn{4}{|c|}{ 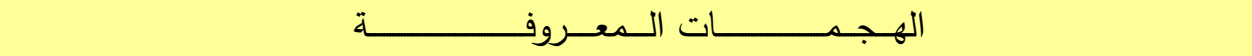 } \\
\hline $\begin{array}{c}\text { Back, land, } \\
\text { Neptune, Pod, } \\
\text { smurf, teardrop }\end{array}$ & $\begin{array}{c}\text { ipsweep, satan, } \\
\text { nmap, } \\
\text { portsweep }\end{array}$ & $\begin{array}{c}\text { ftp_write, } \\
\text { guess_passwd, } \\
\text { warezmaster, } \\
\text { warezclient, } \\
\text { imap, phf, spy, } \\
\text { multihop }\end{array}$ & $\begin{array}{c}\text { Rootkit, } \\
\text { loadmodule, } \\
\text { buffer_overflow, } \\
\text { perl }\end{array}$ \\
\hline \multicolumn{4}{|c|}{ 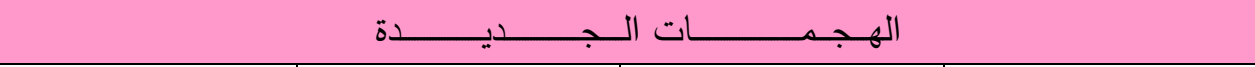 } \\
\hline $\begin{array}{c}\text { Apache2, } \\
\text { udpstorm, } \\
\text { processtable, } \\
\text { mailbomb }\end{array}$ & Saint, mscan & $\begin{array}{c}\text { Named, xlock, } \\
\text { sendmail, xsnoop, } \\
\text { worm, } \\
\text { snmpgeattack, } \\
\text { snmpguess }\end{array}$ & $\begin{array}{l}\text { Xterm, ps, } \\
\text { sqlattack, } \\
\text { httptunnel }\end{array}$ \\
\hline
\end{tabular}

6- خوارزميات مطابقة أنماط السلاسل String Pattern Matching Algorithms:

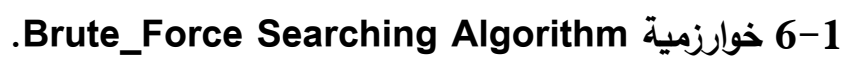
.Knuth - Morris - Pratt String Matching

Boyer-Moore Algorithm خوارزمية

شرحت هذه الخوارزميات وبثكل مفصل ضمن الملحق الخاص بخوارزميات مطابقة الأنماط.

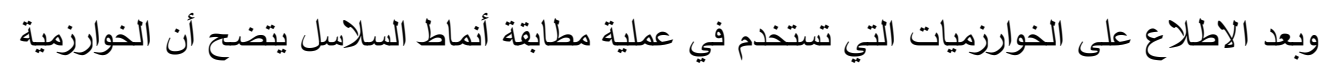

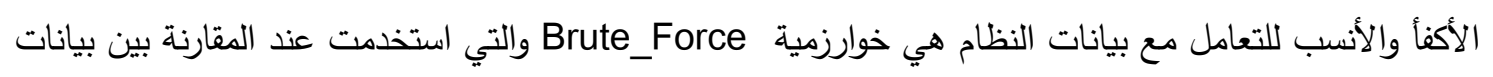


الجداول المستخدمة في النظام المقترح وكما هو موضح في الجدول (2)؛ اذ ان كلتا الخوارزميتين KMP و BM ستحتاج إلى عملية تحليل النمط قبل البدء بعملية البحث بالإضافة إلى حاجة خوارزمية KMP إلى مصنى مصفوفات

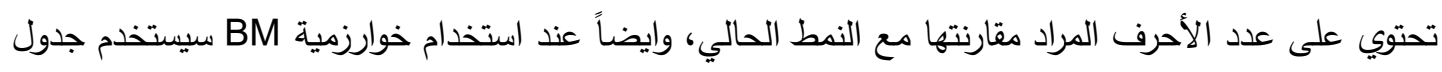
الإزاحة الذي يكون بطول مكافئ لطول السلسلة المراد البحث عنها ضمن النمط، وهذا بدوره سيؤدي إلى زيادة في

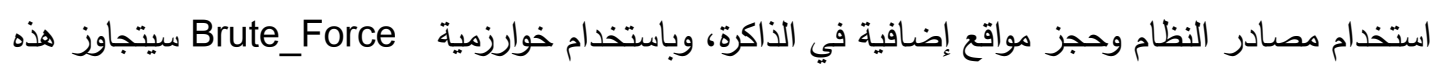

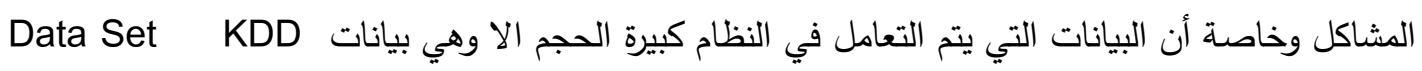
فضلا عن الاستخدام الأمثل لمصادر النظام.

الجدول(2) مقارنة بين خوارزميات مطابقة الأنماط

\begin{tabular}{|c|c|c|c|}
\hline Brute_Force & BM & KMP & الاحتياجات \\
\hline لاتحتاج إلى عملية تحليل النمط & تحتاج إلى عملية تحليل النمط & تحتاج لعملية تحليل النمط & عملية تحليل النمط \\
\hline لا تحتاج إلى مصفوفات & لاتحتاج إلى مصفوفات & تحتاج إلى مصفوفات & الحاجة إلى مصفوفات \\
\hline لا تستخدم جدول الإزاحة & تستخدم جدول الإزاحة & لا تستخدم جدول الإزاحة & استخدام جدول الازاحة \\
\hline استخدام مصادر النظام بكفاءة & زيادة في استخدام مصادر النظام & زيادة في استخدام مصادر النظام & استخدام مصادر النظام \\
\hline
\end{tabular}

7- الخوارزمية المتبعة في Brute_Force Algorithm) Brute_Force):

Input text $T$ of size $n$ and pattern

$P$ of size $m$

$n=T$. length

$m=P$. length

Output starting index of a

Substring of $\boldsymbol{T}$ equal to $\boldsymbol{P}$ or -1

If no such substring exists

For $\mathbf{i}=0$ to $n-m$

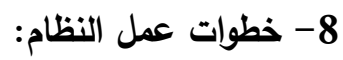

إن تنفيذ النظام اعتمد على خوارزمية مطابقة أنماط السلاسل Brute-force، باستخدام بيئة eclipse، وباعتماد لغة الجافا من خلال الخطوات الآتية:

الخطوة الأولى: قمنا بتهيئة الملفات النصية الخاصة بالعمل والمأخوذة من بيانات KDD، وإنشاء ملفين، الأول

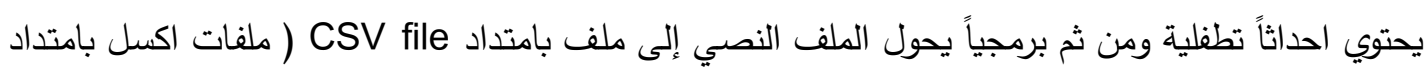

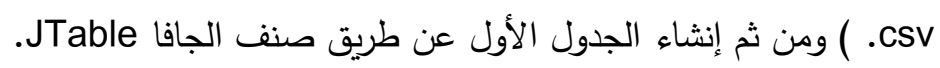
والجدول الثاني سيحوي أحداثاً تطفلية وأحداثاً اعتيادية وأيضاً سيحول إلى ملف ألف csv ومن ثم إنى إلى جدول باستخدام الصنف JTable. 
الخطوة الثالثة: ادخال الجدولين إلى خوارزمية Brute-force لكي تتم عملية المقارنـة وتبيان هل هي احداث تطفليه او طبيعية، لذلك فان خطوات تتفيذ النظام ستكون على النحو الآتي: أ. إذا كانت النتيجة مطابقة (أي بمعنى أن الحدث هو تطفل)، عندئذ سيقوم النظام بعرض النتيجة أسفل الثاشة كلمة intrusion. ب. أما إذا كانت النتيجة غير مطابقة (أي بمعنى أن الحدث هو حدث طبيعي)، عندئذ سيقوم النظام بعرض النفا النتيجة أسفل الثاشة كلمة Normal. ويمكن الاطلاع على الواجهات التنفيذية للنظام من خلال الملحق الخاص بالنتائج.

9_ الاستتتاجات Conclusions

من خلال تصميم الانموذج الأمني وتتفيذه في البحث لغرض كثف التطفل ووفق النتائج التي تم الحصول عليها، نستنتج ما يأتي: 1- بعد الاطلاع على الصيغة المكتوبة بها بيانات KDD، التي ستكون مشابهة لصيغة ملفات CSV، اتضح

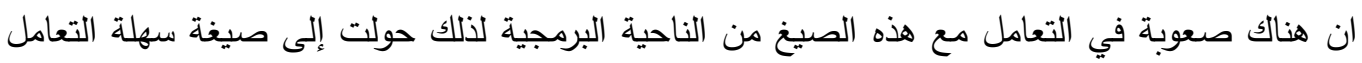

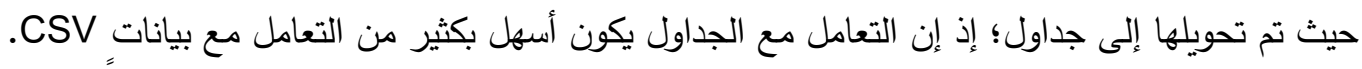

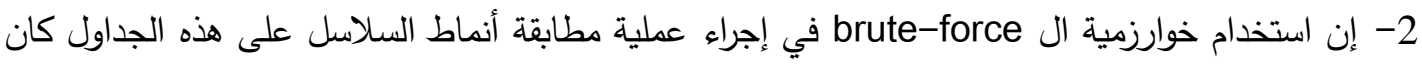

$$
\text { الطريقة الأكفأ للحصول على النتائج وتصنيف الأحداث. }
$$

3- إن استخدام بيئة Eclipse سهل الصعوبات التي واجهت العمل بوصفها بيئة لتنفيذ برامج الجافا؛ إذ إن لها واجهات رسومية غنية بالقوائم والتسهيلات التي يمكن استخدامها في العمل. فضلاً عن أن تكوين جداول النظام تم بطريقة مباشرة باستخدام صنف الجافا (JTable)، دون الحاجة إلى إضافة مكتبات جافا جديدة تستخدم في عملية تصميم الجداول. 
المصادر

[1] د.علاء حسين الحمامي، د.سعد عبد العزيز العاني (2007) ، "تكنولوجيا أمنية المعلومات وأنظمة الحماية "، جامعة عمان الأهلية .

[2] Najla Badie Ibraheem Al-Dabagh, (2006)," Modeling and Implementation of Intrusion Detection Techniques", Ph.D. Thesis, University of Mosul, Computer Sciences .

[3] Adel Sabry Issa, (2009), "A Comparative Study among Several Modified Intrusion Detection System Techniques ", Master Thesis, University of Duhok.

[4] Sahar Lazem Kadoory, (2009), " Design and Implementation of an Embedded Intrusion Detection System (IDS) for Wireless Application", Master Thesis, University of Mosul, Computer Engineering .

[5] Yongro Park, (2005), " A Statistical Process Control Approach for Network Intrusion Detection ", Ph.D. Thesis, The Academic Faculty, Georgia Institute of Technology .

[6] Matti Manninen, (2007), " Using Artificial Intelligence in Intrusion Detection Systems ", Helsinki University of Technology .

[7] DIDS(Distributed Intrusion Detection System)-Motivation, Architecture, and An Early Prototype Snapp (2017), SR; Brentano, J; Dias, G; Goan, TL; Heberlein, LT; Ho, CL; Levitt, KN

[8] Gogoi P, Bhattacharyya DK, Borah B, Kalita K. MLH-IDS (2015), a multi-level hybrid intrusion detection method. The Computer Journal ; 57:602-623.

[9] Wafa' S. Al-Sharafat, Reyadh Sh.Naoum, (2009), "Adaptive Framework for Network Intrusion Detection by Using Genetic-Based Machine Learning Algorithm", Al Al-Bayt University, Information Technology College, Jordan .

[10] Host-based misuse intrusion detection using PCA feature extraction and kNN classification algorithms(2018), Serpen, Gursel*|Aghaei, Ehsan, Electrical Engineering and Computer Science, University of Toledo, Toledo, $\mathrm{OH} 43606$, USA 
[11] Saidat Adebukola Onashoga, Adebayo D. Akinde, and Adesina Simon Sodiya, 2009, "A Strategic Review of Existing Mobile Agent-Based Intrusion Detection Systems", University of Agriculture, Nigeria, Issues in Informing Science and Information Technology Volume 6.

[12] Misuse Detection in a Simulated laaS Environment(2018), Burhan AlBayatiNathan Clarke, Part of the Lecture Notes in Computer Science book series (LNCS, volume 11263)

[13] An Effective Intrusion Detection System Using Flawless Feature Selection, Outlier Detection and Classification(2018), Rajesh Kambattan Kovarasan, Manimegalai Rajkumar, art of the Advances in Intelligent Systems and Computing book series (AISC, volume 713)

[14] Analysis of NSL-KDD Dataset Using K-Means and Canopy Clustering Algorithms Based on Distance Metrics,(2018), H. P. Vinutha, B. Poornima, Part of the Studies in Computational Intelligence book series ( $\mathrm{SCl}$, volume 771)

[15] M. Sabhnani and G. Serpen, (2004), "Why Machine Learning Algorithms Fail in Misuse Detection on KDD Intrusion Detection Data Set", Intelligent Data Analysis, $8(4): 403-415$.

[16] Anithakumari, S,2009, Anithakumari S Dept. of Computer Science \& Engg. Dept. of Computer Science \& Engg. TKM College of Engg. LBSITW, Poojappura Kollam, Kerala Thiruvananthapuram, Kierala $\{\mathrm{IVSL}\}$

[17] Intrusion Detection System Using Pattern Matching Techniques for Wireless Sensor Networks(2018), Jayashree Agarkhed, Gauri Kalnoor Siddarama R. Patil, Part of the Lecture Notes in Networks and Systems book series (LNNS, volume 32)

[18] IEEE Xplore Full-Text HTML : Research and optimization of pattern matching algorithm based on Intrusion Detection System $\{$ IVSL

[19] IEEE Xplore Abstract - A Composite Boyer-Moore Algorithm for the String Matching Problem $\{$ IVSL 


\section{الملحق الخاص بخوارزميات مطابقة الانماط}

\section{Brute_Force Searching Algorithm 1خوارزمية}

تعتبر أكفا الخوارزميات وأبسطها وفكرتها تكون عن طريق مقارنة حرف من النمط مع حرف مع النص، فإذا تطابق الحرفان انتقل إلى الحرف التالي في كل من النمطوالنص. أما في حال لم يتطابقا يحرك مؤشر الحرف في النص إلى الحرف التالي ويرجع مؤشر الحرف في النمط إلى البداية، وسوف تبدأ عملية الدقارنة مرة أخرى. وسوف تستمر عملية هذه إلى أن يتم إيجاد التطابق في كل حروف النمط ، أو الوصول لنهاية النص. وسيشار إلى طول النمط بالحرف M أما طول النص سوف يتم الاشارة له بالحرف N. وسيتوقف البحث عندما نصل ل N-M؛ لأنه عند الوصول إلى تلك الخانة وحتى إذا كان الحرف التالي منطابقاً يتم التوقف؛ لأن النص سوف يكون أصغر من النمط.

الثكل (1) يوضح عمليه البحث عن النمط needle، إذ قورن الحرف الأول في النمط مع الحرف الأول

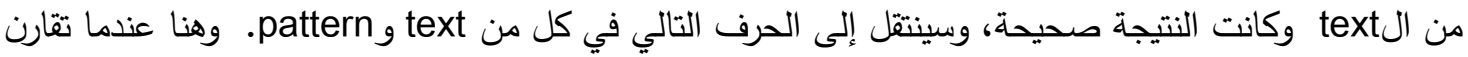
فالنتيجة غير صحيحة وبالتالي يحرك مؤشر النص إلى الأمام، ويرجع النمط إلى لبداية، وتبدأ عملية البحث مرة

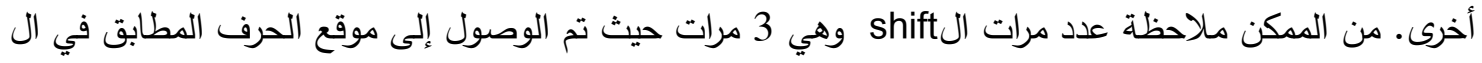
• ثلاثة [16 [ndex

\section{$h$ a y $n$ e e d s a n n e ed I e $x$}

n e e d I e

n e e d I e

$\mathbf{n}$ e e d I e

n e e d I e

$$
\begin{aligned}
& \mathbf{n} \text { e e d I e } \\
& \mathbf{n} \text { e e d I e } \\
& \mathbf{n} \text { e e d I e } \\
& \mathbf{n} \text { e e d I e } \\
& \mathbf{n} \text { e e d I e } \\
& \mathbf{n} \mathbf{e} \text { ed I e } \\
& \mathbf{n} \text { e e d I e }
\end{aligned}
$$

Brute_Force Search الثكل (1) البحث عن نمط معين باستخدام خوارزمية ال تقوم هذه الخوارزمية بمقارنة كل حرف مع الحرف الآخر ، وفي حال لم يتطابقا ستعاد عملية المقارنة

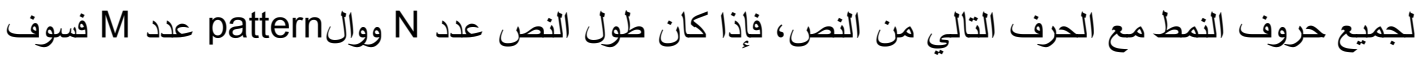

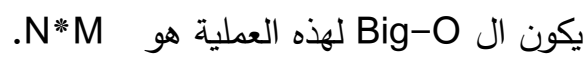




\section{:Knuth - Morris - Pratt String Matching خوارزمية}

في عام 1977 نشر الثلاثي Knuth (صاحب كتاب Art of programming الثهير) و و Pratt مقالة Fast Pattern Matching in Strings وتحدثوا فيها عن ايجاد خوارزمية أسرع من الطريقة التي تعتمد على Brute-Force وسميت هذه الخوارزمية باسم هؤلاء الأشخاص واختصاراً KMP.

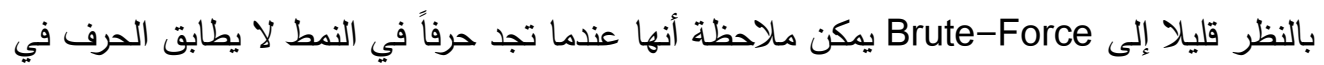

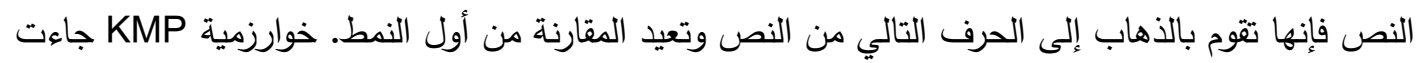
لتحسين تلك الخوارزمية حيث تم التخلص من عمليات المقارنة المتكررة، طريقه عملها على النحو الاتي:

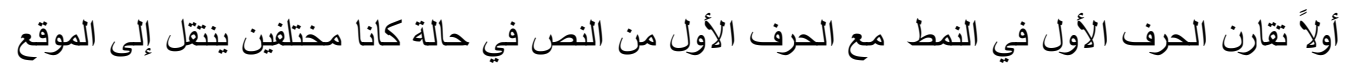

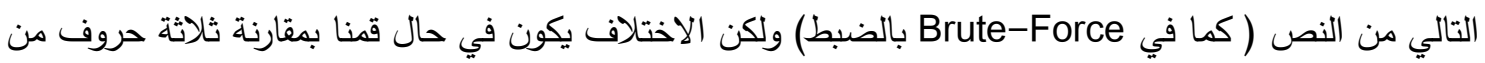

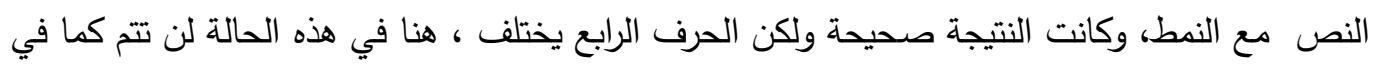

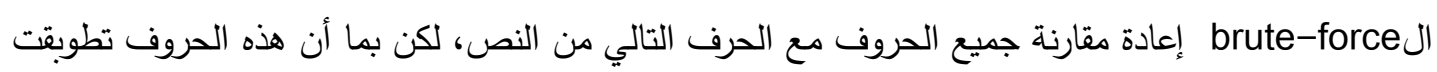

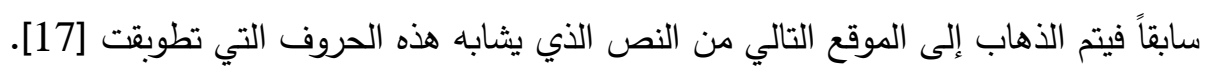

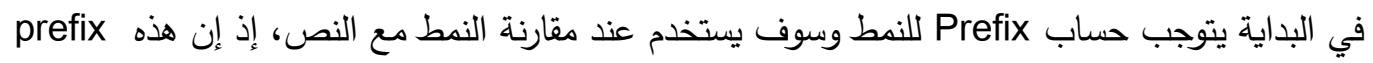

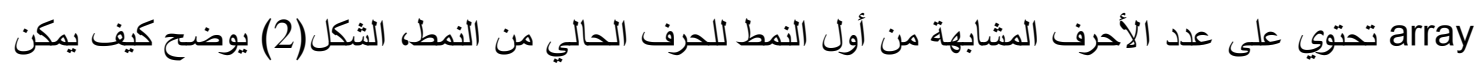
حسابه:

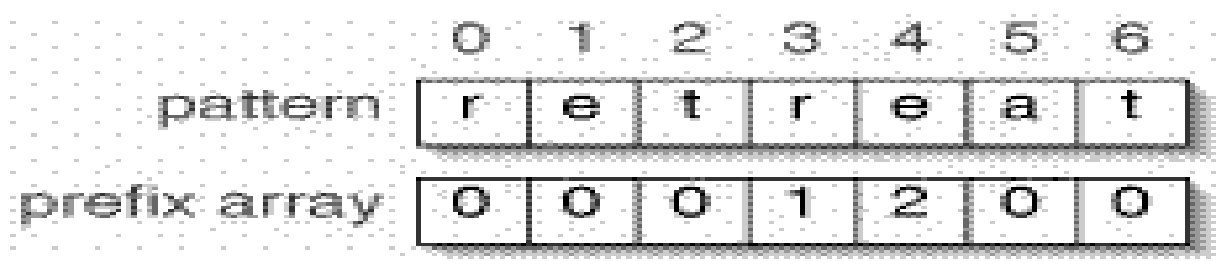

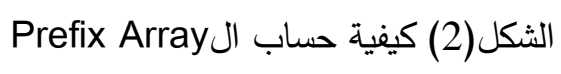

يمكن ملاحظة الموقع 3 (الحرف r) يحتوي على 1 والسبب أن الحرف r موجود في الموقع 0. 1 تثير إلى عدد الأحرف الprefix ـ أيضاً الموقع التالي 4 (الحرف e) يحتوي على 2 والسبب أن الحرفين في الموقعين

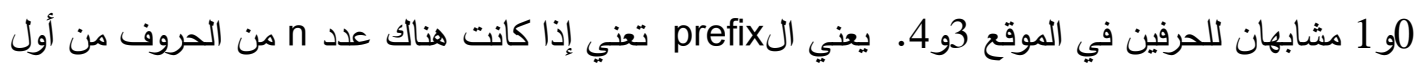

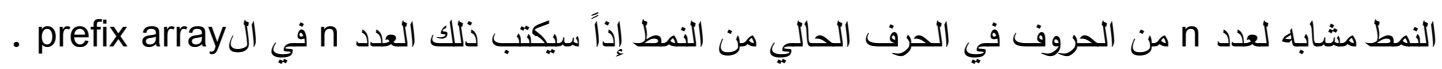
مثال أخر كما في الثكل(3): 


\section{2}

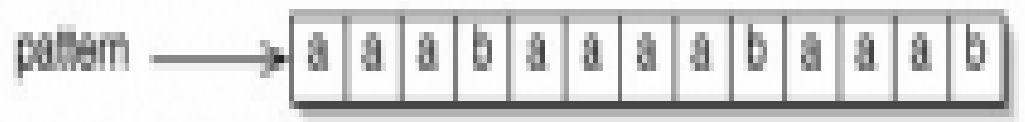

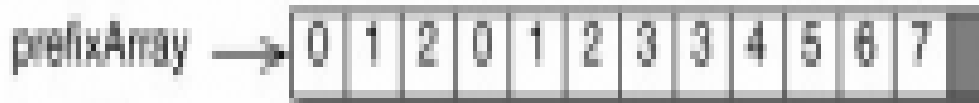

$1=12$

mithes $=7$

Prefix Array الثكل(3) كيفية حساب الP

كما هو موضح مسبقاً فإن هذه الprefix array سيستخدم في خوارزميه البحث عندما لا يتطابق الحرف

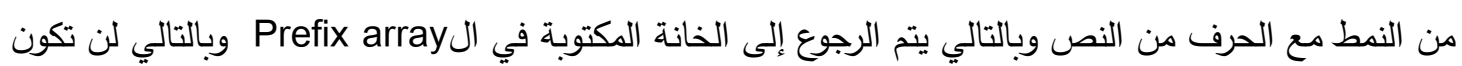
هناك عملية المقارنة للأحرف التي أجريت عملية المقارنة عليها [16].

الثكل(4) يوضح كيفية البحث عن النمط في النص:

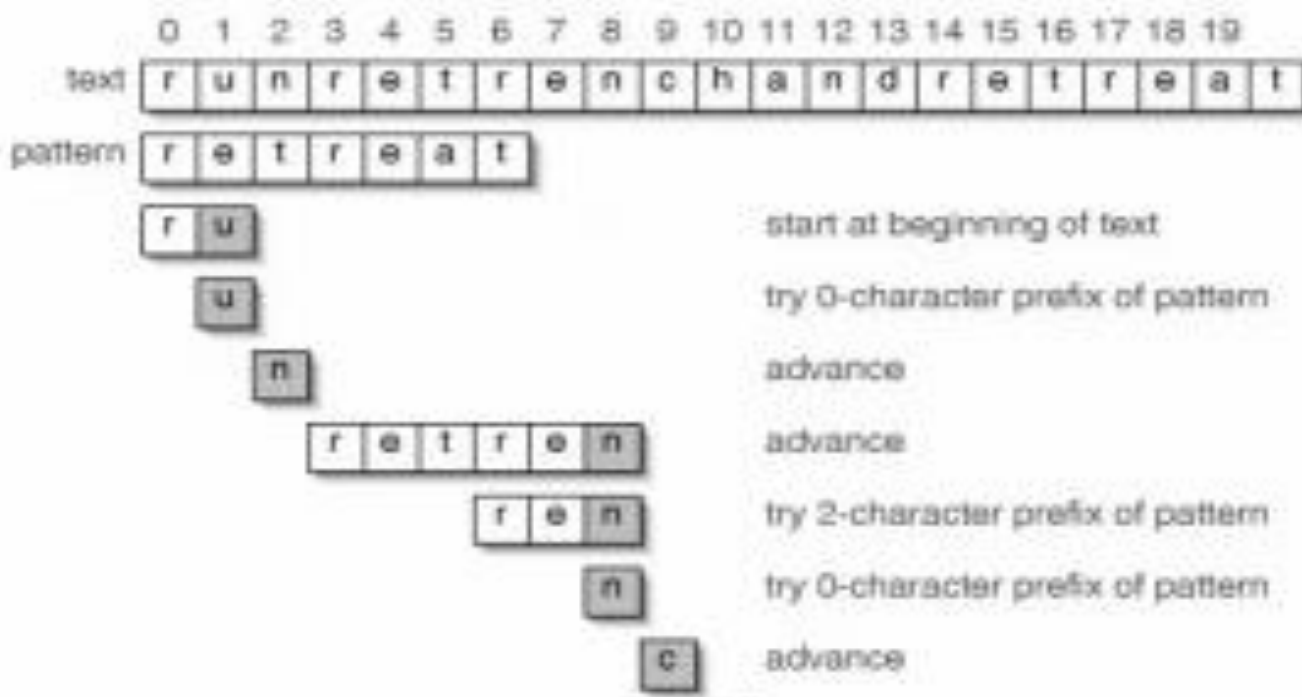

h

a

n

(D)

\begin{tabular}{|l|l|l|l|l|l|l|}
\hline$t$ & 0 & $\mathrm{t}$ & $\mathrm{t}$ & $\boldsymbol{e}$ & $\mathrm{a}$ & $\mathrm{t}$ \\
\hline
\end{tabular}

الثكل(4) كيفية البحث عن النمط في النص الأصلي 
في هذه الخوارزمية ، في البدء سيقارن الحرف الأول من النمط مع الحرف الأول من النص، عندما يكونان متطابقين، يتم الذهاب إلى الحرف التالي في كل من النمط والنص. تجرى عملية المقارنة وسيلاحظ أن الحرفين غير متطابقين، إذاً في هذه الحالة سوف يكون الرقم الموجود في الخانة الأولى في

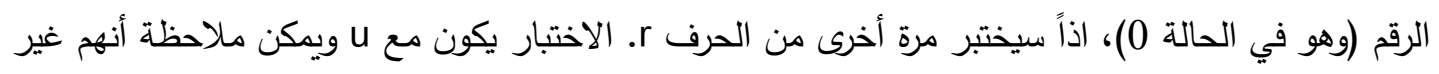

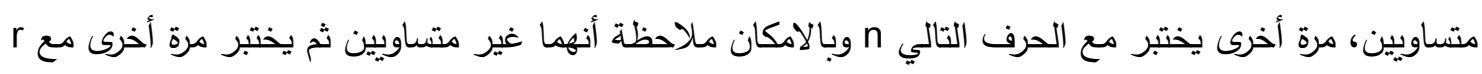

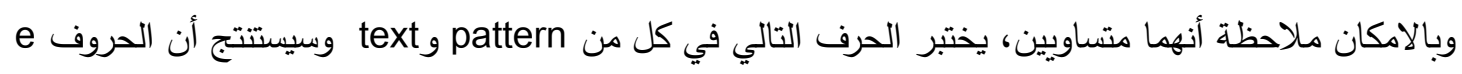

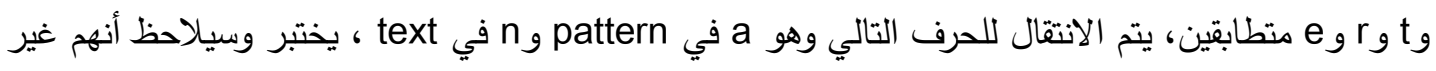

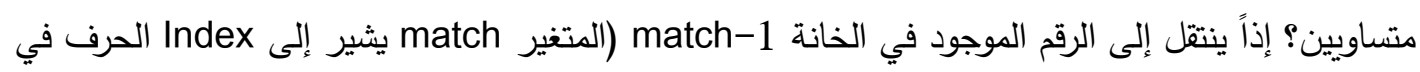

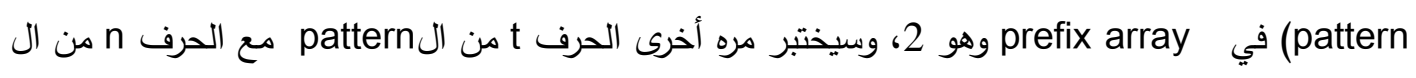
S سيلاحظ أنهم غير متطابقين، ينتقل الآن إلى الخانة prext

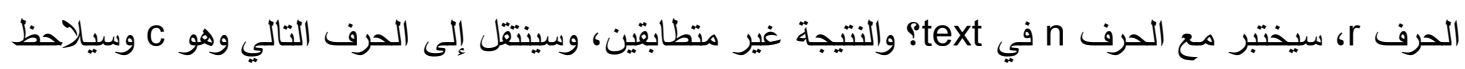

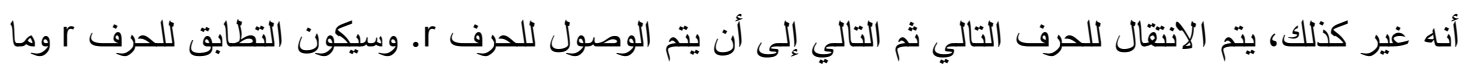

\section{3-3و خوارزمية Boyer-Moore Algorithm}

تعد هذه الخوارزمية إحدى أسرع الخوارزميات المستخدمة في عمليه البحث عن النصوص وسميت بذلك

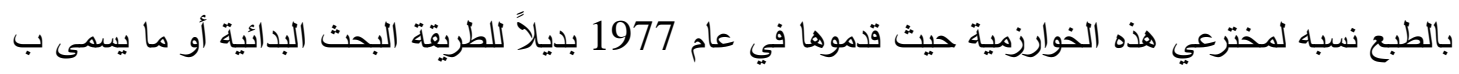
.Naive Searching

المقارنة في هذه الخوارزمية تعتمد على البدء من اليمين إلى اليسار وليس كما هو الحال مع الخوارزميات

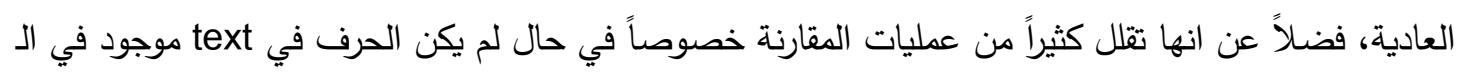
pattern

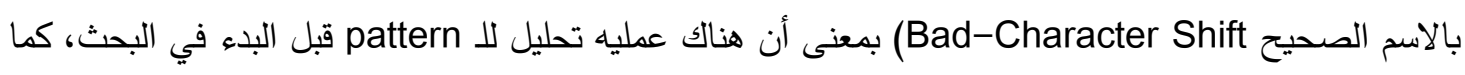

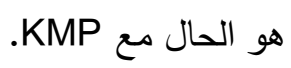
جدول الإزاحة يجب أن يكون حجمه مساوياً لحجم ال character set التي سيتكون منها النص text والنمط pattern، وبما أنه حاليا سيبيحث عن الحروف الإنجليزية سوف يكون حجم الجدول بعدد حروف ASCII

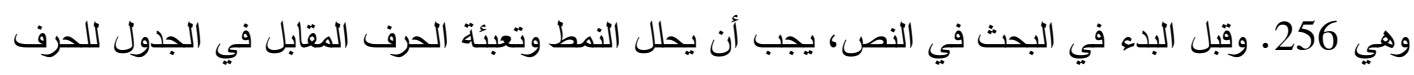
المقابل للنمط بمقدار ظهور آخر index للنمط.

E، D، C، B،A مكونه من الحروف character set المثال التالي يوضح طريقة البحث، فإذا كانت

والنمط هو DECADE، سوف يكون شكل الجدول كما في الثكل(5) 


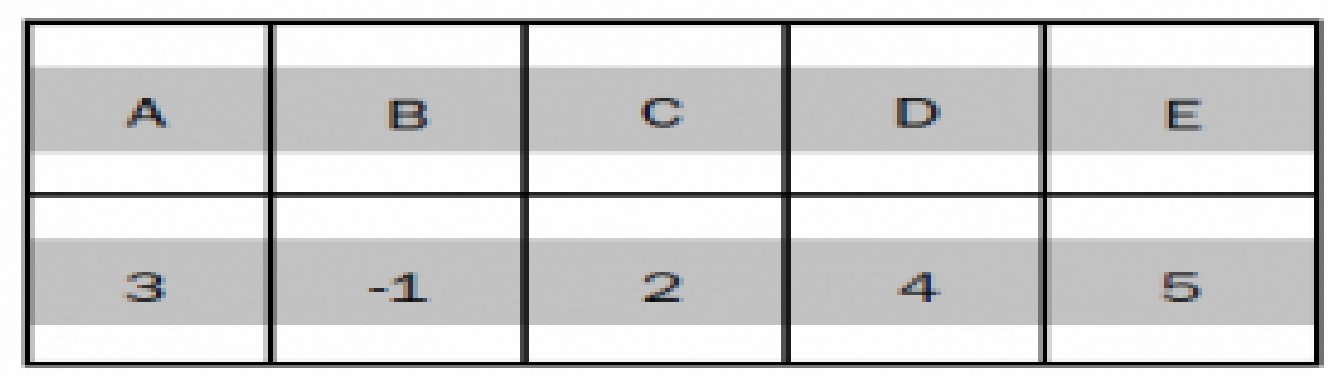

$$
\text { الثكل(5) جدول الإزاحة }
$$

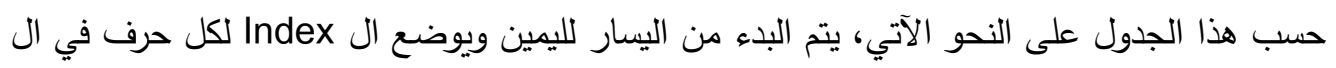

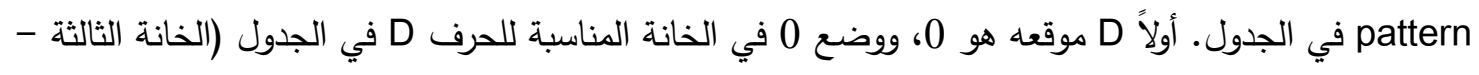
الترقيم من 0-). ثانياً الحرف E موقعه هو 1 ووضع 1 في الخانة المناسبة للحروف E في الجدول. العملية. يمكن ملاحظة أن الحرف D سيتكرر مرة ثانية وسيكتب الIndex الجديد (4) في الخانة القديمة، وبنفس الأمر للحرف E. وهذا هو أساس عملية التحليل في البداية، فقط المطلوب موقع آخر ظهور للحرف، وهذا هو المطلوب في عملية البحث في حال اختلف الحرف من النص مع النمط. يمكن أن تكون بقية الحروف في ال character set التي لا توجد لها قيم وسيوضع

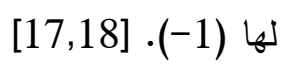
طريقه البحث في خوارزميه Boyer-Moore تكون كما توضح بالثكل(6):

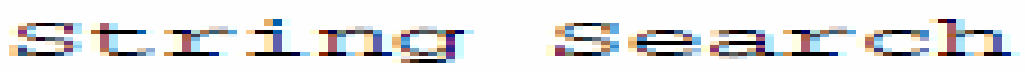
I고프

I스IDg 파 츠모

\section{포 츠}

الشكل(6) طريقة البحث في خوارزميةBM

أولاً البحث كما ذكر في هذه الخوارزمية سوف يكون من اليمين لليسار بمعنى أول حرف سوف يتم البدء

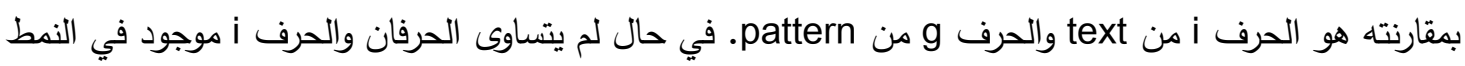
سوف يحرك النمط حرفين إلى الأمام وهكذا يتطابق i مع أ. وهكذا تطابقت الكلمتان.

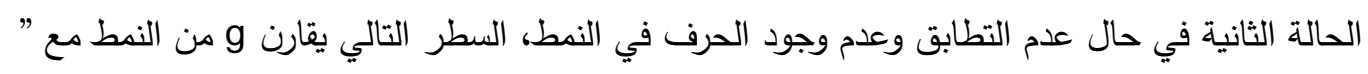
" مسافة من text، وبما أنهم لا يتطابقان والحرف ” ” لا يوجد في النمط سوف يحرك النمط بعدد حروف النمط وهي 4 في هذه الحالة [19]. يمكن النظر للشكل(7) يبين طريقة البحث في خوارزمية BM وهي تبين كيف يتم البحث بشكل أوضح 


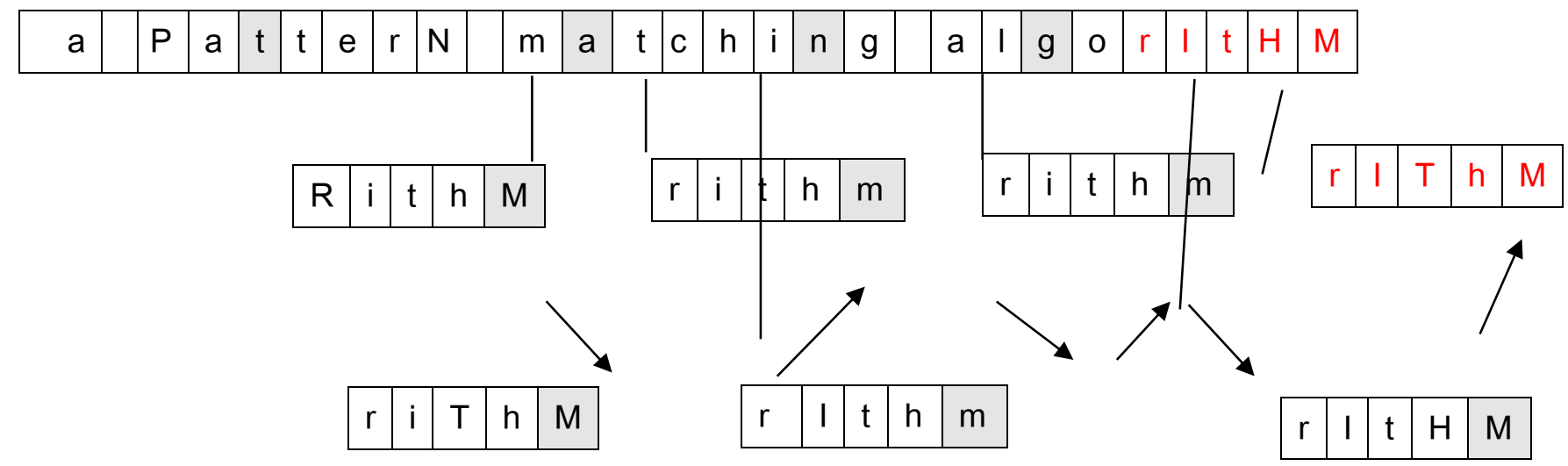

BM اللثكل(7) طريقة البحث في خوارزمية

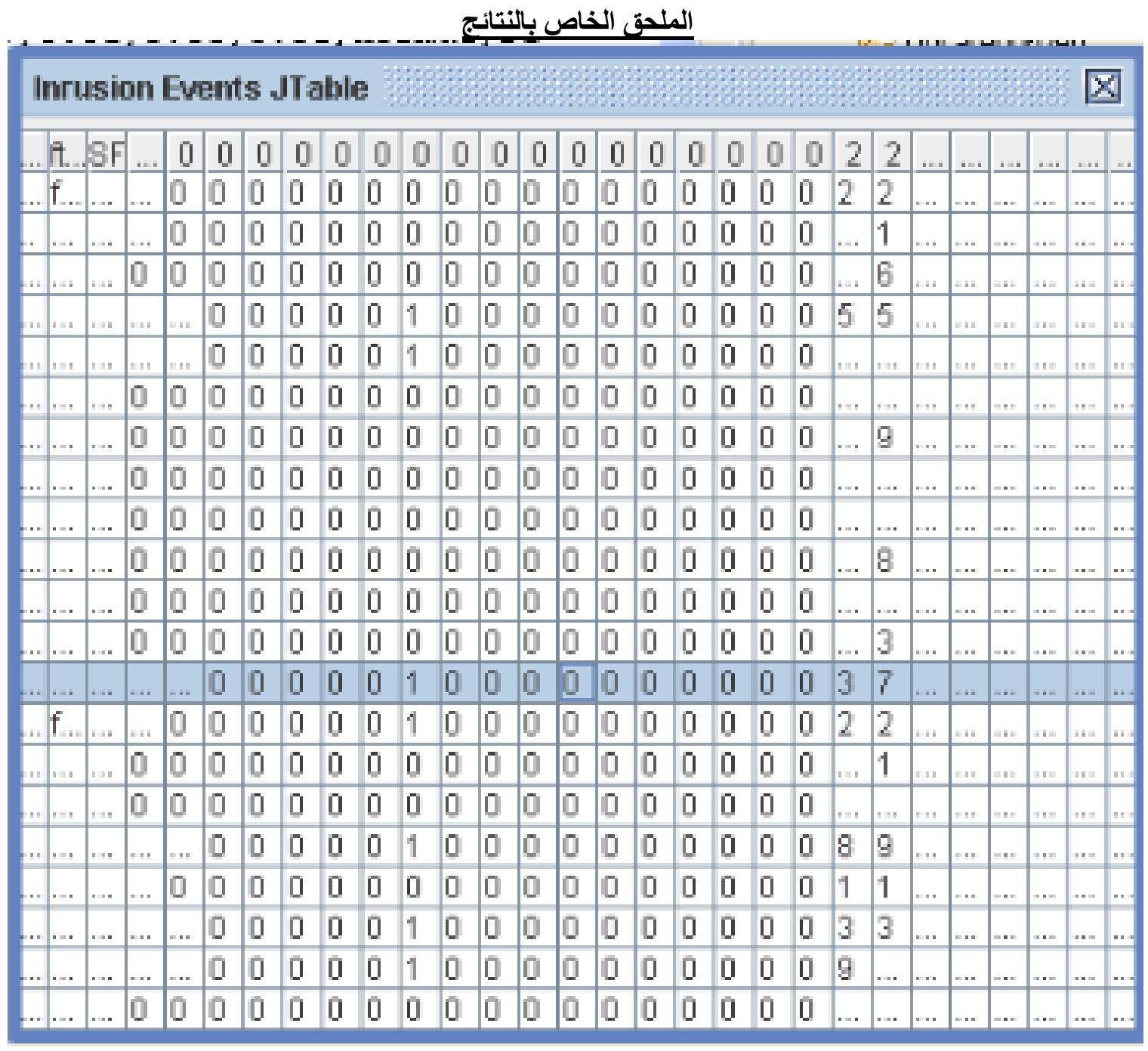

الثكل (1) جدول يحتوي على أحداث تطفلية 
Our Events JTable $600 \%$ W

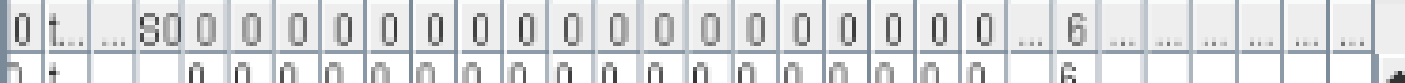

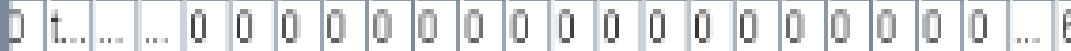

$\begin{array}{llllllllllllllllllllllllllllll}0 & t & \ldots & \ldots & \ldots & 0 & 0 & 0 & 0 & 0 & 0 & 0 & 0 & 0 & 0 & 0 & 0 & 0 & 0 & 0 & 0 & 0 & 0 & \ldots & \ldots & \ldots & \ldots & \ldots & \ldots & \ldots\end{array}$

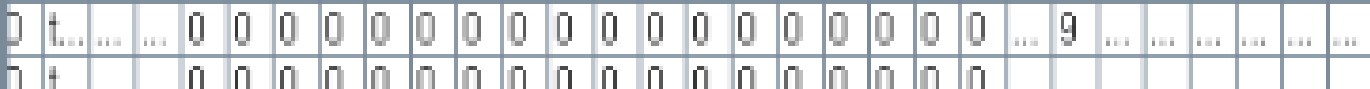

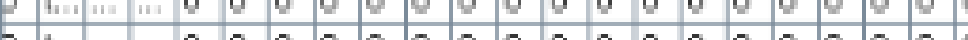

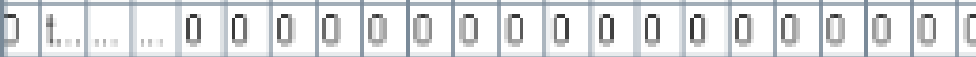

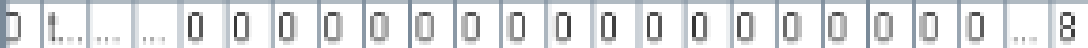
$\begin{array}{llllllllllllllllllllllll}0 & 1 . . . . & \ldots & 0 & 0 & 0 & 0 & 0 & 0 & 0 & 0 & 0 & 0 & 0 & 0 & 0 & 0 & 0 & 0 & 0 & 0\end{array}$

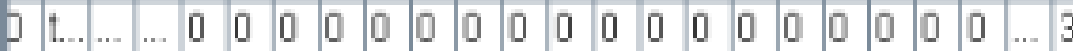

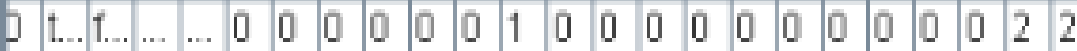

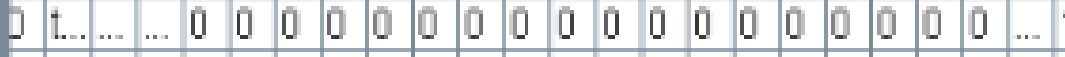

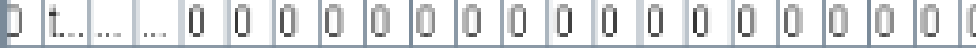

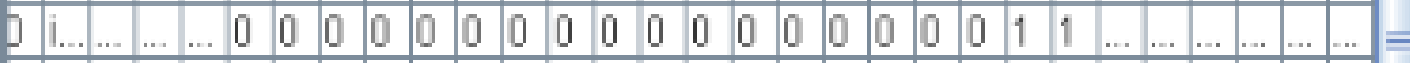
\begin{tabular}{llllllllllllllllllllllllllllllll}
0 & $t$ & $\ldots$ &.. & $\ldots$ & 0 & 0 & 0 & 0 & 0 & 0 & 0 & 0 & 0 & 0 & 0 & 0 & 0 & 0 & 0 & 0 & 0 & 0 & $\ldots$. & $\ldots$ & $\ldots$ & $\ldots$ & $\ldots$ & $\ldots$ & $\ldots$ & $\ldots$ \\
\hline
\end{tabular} $\begin{array}{llllllllllllllllllllll}0 & t_{i} & : & . & 0 & 0 & 0 & 0 & 0 & 0 & 0 & 0 & 0 & 0 & 0 & 0 & 0 & 0 & 0 & 0 & 0 & 0\end{array}$ $\begin{array}{llllllllllllllllllllll}0 & t_{1} & \ldots & \ldots & 0 & 0 & 0 & 0 & 0 & 0 & 0 & 0 & 0 & 0 & 0 & 0 & 0 & 0 & 0 & 0 & 0 & 0\end{array}$ $\begin{array}{lllllllllllllllllllllllll}0 & t_{1 . .} & \ldots & \ldots & 0 & 0 & 0 & 0 & 0 & 0 & 0 & 0 & 0 & 0 & 0 & 0 & 0 & 0 & 0 & 0 & 0 & 0 & \ldots & 7\end{array}$

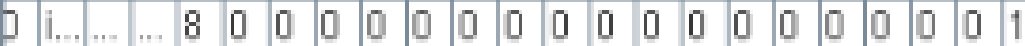

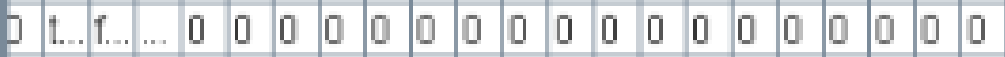

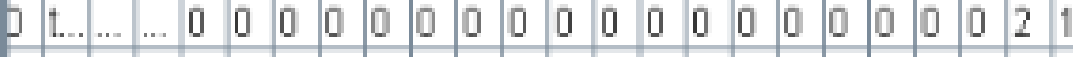

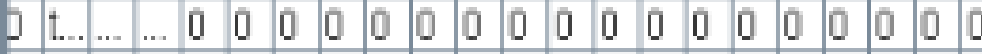

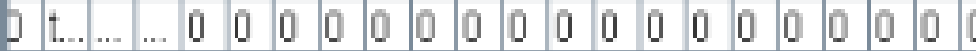

الثكل (2) جدول يحتوي على احداث تطفلية واعتيادية 


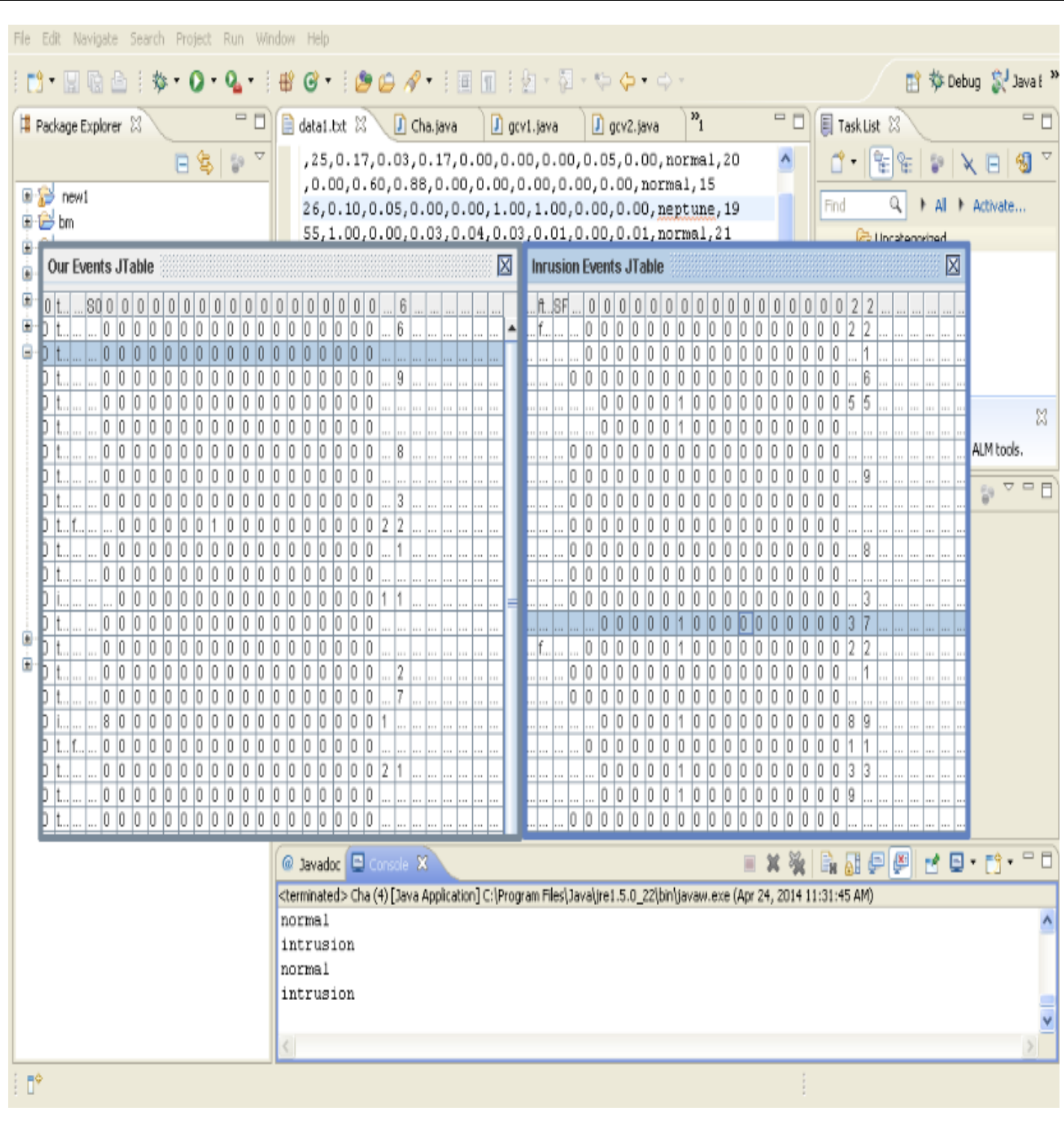

الثكل (3) عملية مقارنة الجدولين

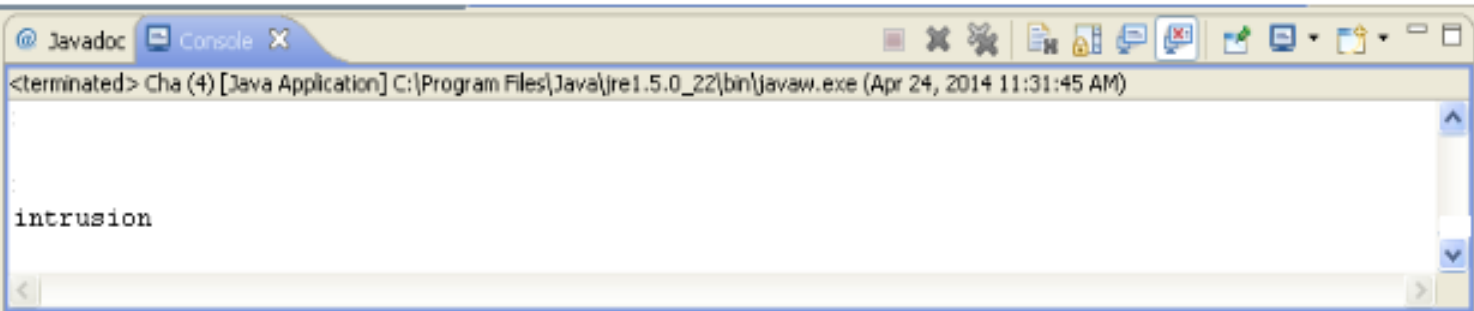

الثكل (4) نتيجة المقارنة المطابقة

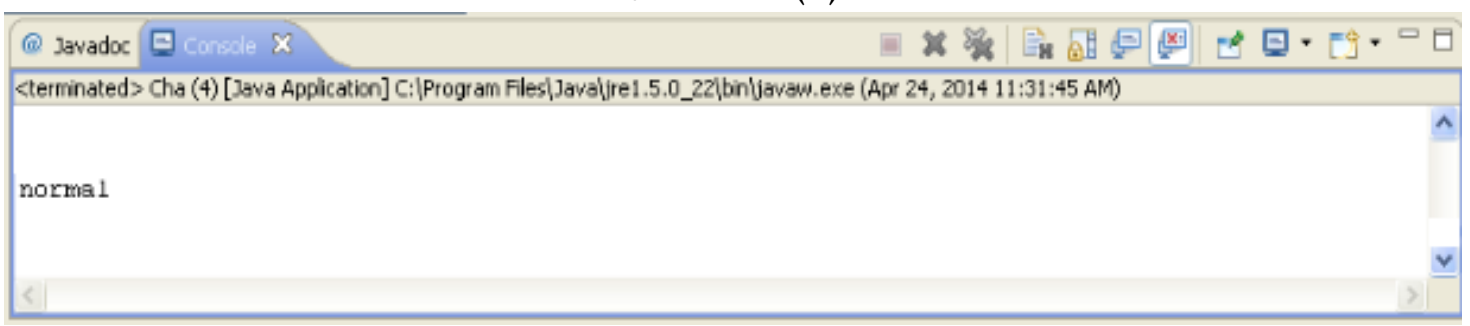

الثكل (5) نتيجة المقارنة الغير المطابقة 